\title{
Spontaneous Peripheral Ameloblastic Odontoma in a Male Sprague-Dawley Rat
}

\author{
Yinghua Li', Han-Ik Bae², Hak-Soo Kim ${ }^{1}$, Min-Soo Kang ${ }^{1}$, Bo-Ho Gong' ${ }^{1}$, Won-Hee Jung ${ }^{1}$, Sranna Lee ${ }^{1}$, \\ Jin-Sook Bae ', Kap-Ho Kim ${ }^{1}$, Si-Whan Song', Jae-Hyun Lee' and Boo-Hyon Kang' \\ ${ }^{1}$ Department of Pathology, Chemon Inc., Yongin, Korea \\ ${ }^{2}$ Department of Pathology, Kyungpook National University Medical Center, Daegu, Korea
}

(Received November 22, 2016; Revised February 2, 2017; Accepted February 21, 2017)

\begin{abstract}
Peripheral ameloblastic odontoma is a rare variant of odontogenic tumor occurring in the extraosseous region. The present report describes a spontaneous tumor in male Sprague-Dawley (SD) rats. The clinically confirmed nodule in the right mandibular region was first observed when the rat was 42 weeks and remained until the terminal sacrifice date when the animal was 48 weeks of age. At necropsy, a well demarcated nodule, approximately $2.5 \times 2.0 \times 2.0 \mathrm{~cm}$, protruded from the ventral area of the right mandible. The nodule was not attached to mandibular bone and was not continuous with the normal teeth. Histopathologically, the tumor was characterized by the simultaneous occurrence of an ameloblastomatous component and composite odontoma-like elements within the same tumor. The epithelial portion formed islands or cords resembling the follicle or plexiform pattern typical of ameloblastoma and was surrounded by mesenchymal tissue. Formation of eosinophilic and basophilic hard tissue matrix (dentin and enamel) resembling odontoma was observed in the center of the tumor. Mitotic figures were rare, and areas of cystic degeneration were present. Immunohistochemically, the epithelial component was positive for cytokeratin AE1/AE3 (CK AE1/AE3), and the mesenchymal component and odontoblast-like cells were positive for vimentin, in the same manner as in normal teeth. On the basis of these findings, the tumor was diagnosed as a peripheral ameloblastic odontoma in an extraosseous mandibular region in a SD rat. In the present study, we report the uncommon spontaneous peripheral ameloblastic odontoma in the SD rat. We also discuss here the morphological characteristics, origin, histochemical, and immunohistochemical features for the diagnosis of this tumor.
\end{abstract}

Key words: Ameloblastoma, Odontogenic ectomesenchyme, Odontoma, Peripheral ameloblastic odontoma, Sprague-Dawley rat

\section{INTRODUCTION}

The ameloblastic odontoma is an extremely uncommon odontogenic tumor, and is defined by World Health Organization (WHO) as: "A neoplasm that includes odontogenic ectomesenchyme in addition to odontogenic epithelium that resembles an ameloblastoma in both structure and behavior. Because of the presence of odontogenic ectomesen-

Correspondence to: Yinghua Li and Boo-Hyon Kang, Department of Pathology, Chemon Inc., 240, Nampyeong-ro, Yangji-myeon, Cheoin-gu, Yongin-si, Gyeonggi-do 17162, Korea

E-mail: l-iyinghua@hanmail.net (Yinghua Li), bkang51@chemon.co.kr (Boo-Hyon Kang)

This is an Open-Access article distributed under the terms of the Creative Commons Attribution Non-Commercial License (http:// creativecommons.org/licenses/by-nc/3.0) which permits unrestricted non-commercial use, distribution, and reproduction in any medium, provided the original work is properly cited. chyme, inductive changes take place leading to the formation of dentin and enamel in part of the tumor" (1).

The ameloblastic odontoma was described as a mixed odontogenic tumor originating from proliferating epithelial and mesenchymal components characterized by simultaneous occurrence of ameloblastoma and composite odontoma within the same tumor. Odontogenic epithelium was described to resemble that of an ameloblastoma with the additional features of odontogenic ectomesenchyme and induction of mineralized dental tissue resembling that of composite odontoma (2-4).

Ameloblastic odontoma has been reported in a variety of mammalian species, including dog (5), cat (6), cow (7), sheep (8), llama (9), non-human primate $(10,11)$, and rat $(2,12,13)$.

Peripheral odontogenic tumor is an uncommon, extraosseous and odontogenic tumor in soft tissue and it was first reported by Stanley and Krogh in 1959 (14). Peripheral 
odontogenic tumor is histologically indistinguishable from the typical intraosseous odontogenic tumor but is different in that it occurs in the soft tissue overlying alveolar bone but is separate from it $(15,16)$.

The etiology of peripheral odontogenic tumor is unclear. The tumor can arise from the extraosseous epithelial remnant of the dental lamina or the basal cell layer of the oral mucosa, which are believed to have odontogenic potential $(17,18)$.

In this study, we report peripheral ameloblastic odontoma that resembled previously reported uncommon tumors histologically similar to ameloblastic odontoma. The purpose of this case report is to describe the morphological features, origin, histochemical, and immunohistochemical characteristics of peripheral ameloblastic odontoma observed as an incidental finding in a male SD rat during a chronic toxicity study.

\section{MATERIALS AND METHODS}

Experimental animal and animal husbandry. The animal was a male specific pathogen free (SPF) Sprague Dawley (Hsd; SD) rat purchased from Koatech Inc (Pyeongtaek, Korea). It was used as a vehicle control animal in the 8-week recovery group in a 32-week repeated-dose toxicity study and was submitted for sacrifice at the end of the study. The animal was housed in a solid-bottom polycarbonate cage under routine controlled conditions (temperature, $23 \pm 3^{\circ} \mathrm{C}$; humidity, $55 \% \pm 15 \%$; lighting, $12 \mathrm{hrs)} \mathrm{and}$ had free access to Teklad-certified, irradiated, global $18 \%$ protein rodent diet 2918C (Harlan Laboratories, Inc., IN, USA) and sterilized tap water. This animal was housed in a facility (Chemon Inc., Yongin, Korea) approved by the Association for Assessment and Accreditation of Laboratory Animal Care International (AAALAC). This study was also approved by the Animal Experiment Committee of Chemon Inc. based on the Animal Protection Act. The study was compliant with Good Laboratory Practice (GLP) standards. The animal's clinical signs were observed once a day.

Necropsy and histopathological evaluation. The animal was euthanized by exsanguination via the abdominal aorta under isoflurane (Ifran liquid, Hana Pharm. Co., Ltd., Seoul, Korea) anesthesia and necropsied under the supervision of a pathologist. The partial mass was fixed in $10 \%$ neutral buffered formalin followed by paraffin embedding. Additionally, the partial mass and normal incisor teeth were decalcified in $10 \%$ formic acid solution for 7 day at room temperature before paraffin embedding. After routine tissue processing, tissue sections were cut to approximately $4 \mu \mathrm{m}$ thickness, stained with hematoxylin and eosin (H\&E). Additionally, undecalcified sections from the tumor mass were stained with von Kossa for calcified tissue and tartrate-resistant acid phosphatase (TRAP) for visualization of osteoclasts (counterstaining with methyl green). The decal- cified mass sections and normal teeth were stained with Masson's Trichrome for collagen and with the periodic acid-Schiff (PAS) reaction for glycogen.

Immunohistochemical analysis. Immunohistochemical studies were performed on the tumor sections and normal teeth using a biotin-avidin peroxidase complex method and an automated immunostainer (BenchMark ${ }^{\circledR}$; Ventana Medical Systems, Inc., Tucson, AZ, USA). The primary antibodies (prediluted) were used cytokeratin AE1/AE3 (CK AE1/ AE3; mouse monoclonal, Signet, Seongnam, Korea), and vimentin (mouse monoclonal, Signet). An indirect biotinstreptavidin system (iView DAB Detection Kit; Ventana Medical Systems, Inc.) was used as the secondary antibody. The tumor sections were counterstained with hematoxylin (Ventana Medical Systems, Inc.) and then treated with Bluing Reagent (Ventana Medical Systems, Inc.). The semiquantitative scoring system categorized severity as minimal for fewer than 5\% tissue stained, slight for 5\% 25\%, moderate for $25 \% \sim 50 \%$, marked for $50 \% \sim 75 \%$, and severe for more than $75 \%$ tissue stained. An additional category of negative representing absence of the tissue was stained.

\section{RESULTS}

As a clinical sign, the nodule was observed in the right mandibular region from 42 weeks of age until 48 weeks of age, when the animal was sacrificed. The other physical examinations were unremarkable in this case.

At necropsy, a well demarcated nodule, approximately $2.5 \times 2.0 \times 2.0 \mathrm{~cm}$, protruded from the ventral area of the right mandible. The nodule was dissected free by blunt dissection with relative ease and was totally removed. It was not attached to the mandibular bone or continuous with the

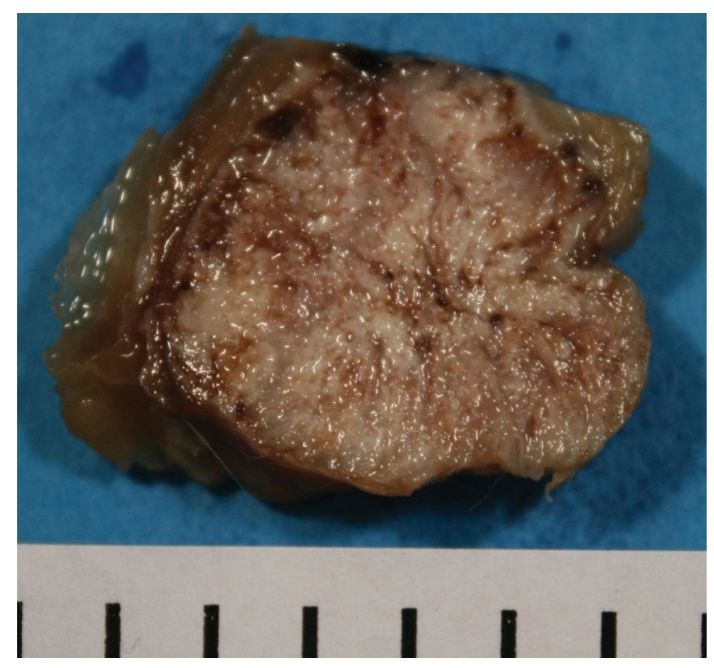

Fig. 1. Gross appearance. The cut surface of the tumor appeared whitish after fixation. 1 scale $=0.5 \mathrm{~cm}$. 

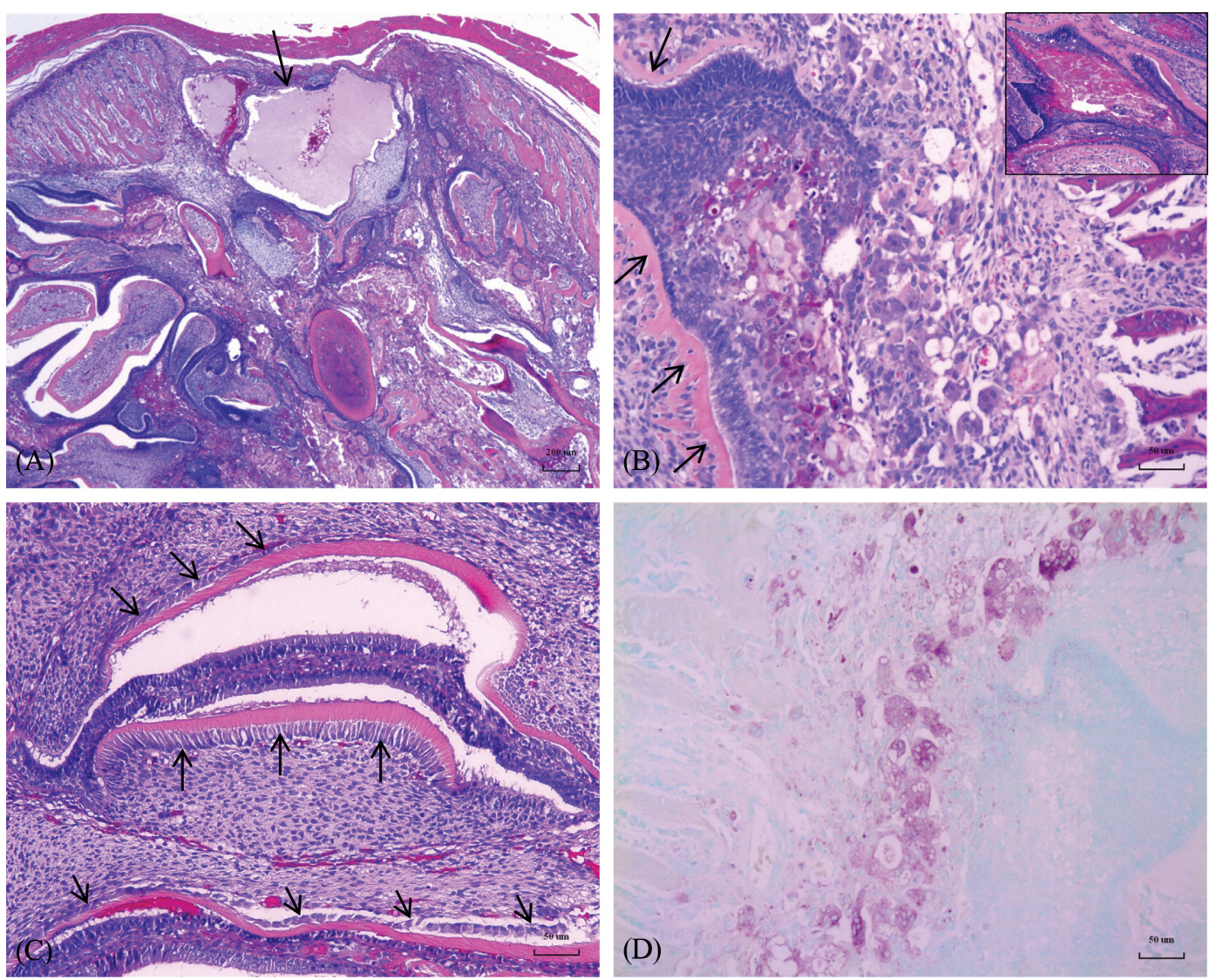

Fig. 2. (A) H\&E staining. Odontogenic epithelial cells were arranged in cords or follicular patterns surrounded by mesenchymal cells. The ameloblast-like cells were palisaded in the peripheral area and the stellate reticulum-like cells were arranged in central area. Areas of cystic degeneration were present also (arrow). Scale bar $=200 \mu \mathrm{m}$. (B) H\&E staining. The epithelial component contained eosinophilic granular cells at its center. Multinucleated polygonal cells were present along the surface of osseous tissue and eosinophilic material (arrows) was layered between the epithelium and the mesenchymal stroma. Scale bar $=50 \mu \mathrm{m}$. Inset: areas of squamous metaplasia surrounded by an ameloblast-like cells. (C) H\&E staining. The abortive tooth structures consisted of a pulp cavity lined by a layer of ameloblast-like cells, dentin, and single layer of cuboidal to flattened odontoblast-like cells (arrows). Scale bar $=50 \mu \mathrm{m}$. (D) TRAP staining. The many TRAP-positive cells (purple cytoplasm) were multinucleated osteoclasts. Scale bar $=50 \mu \mathrm{m}$.

normal teeth. Further, the nodule was not adherent to either the salivary gland or duct. After fixation, the cut surface of the nodule was whitish with friable appearance (Fig. 1). The surface oral mucosa was not ulcerated and appeared to be normal. All other organs and tissues were normal.

Histopathologically, the tumor was surrounded by bonelike tissue and loose fibrous connective tissue and had a biphasic pattern of epithelial and mesenchymal components. Much of the periphery of the mass was immature, similar to ameloblastoma. The central area of the tumor resembled composite odontoma. The odontogenic epithelium formed nests and islands that were interspersed within the loose mesenchymal tissue resembling dental pulp. Mesenchymal components consisted of immature fibrous tissues comprising poorly differentiated spindles of stellate cells (Fig. 2A). Odontogenic epithelial cells were arranged in cords or follicular patterns surrounded by condensed mesenchymal cells (Fig. 2A). Columnar ameloblast-like cells arranged in palisades were present in the outer areas of the dentin structure. There were stellate reticulum-like cells present in the central area of ameloblast-like cells. Eosinophilic material containing osteoblast-like small cells, presumably osteodentin, was frequently observed between the epithelial layer and its circumscribing mesenchymal stroma (Fig. 2B). Some odontogenic epithelial islands consisted of stellate reticulum-like cells with coarse, granular eosinophilic cytoplasm and areas of squamous metaplasia surrounded by a layer of columnar ameloblast-like cells with well-polarized nuclei (Fig. 2B). In some areas, inverted or cup-shaped abortive teeth structures were present, consisting of layers of ameloblast-like cells, dentin, and single layer of cuboidal to flattened odontoblast-like cells all lining a pulp cavity (Fig. 2C). Many TRAP-positive polygonal cells were observed along the osseous tissue surface. These cells had multiple nuclei with rather deeply stained purple cytoplasm (Fig. 2D), consistent with osteoclasts. 
Formation of eosinophilic or basophilic hard tissue matrix was observed in the center of tumor. The hard tissues that were stained basophilic were considered to show advanced calcification (Fig. 3A), confirmed by positive von Kossa staining (Fig. 3B). The hard tissue that were stained eosinophilic was considered to show hypocalcification (Fig. 3A), confirmed by negative von Kossa staining (Fig. 3B). Therefore, calcification of dentin may be irregular (Fig. 3A, 3B).
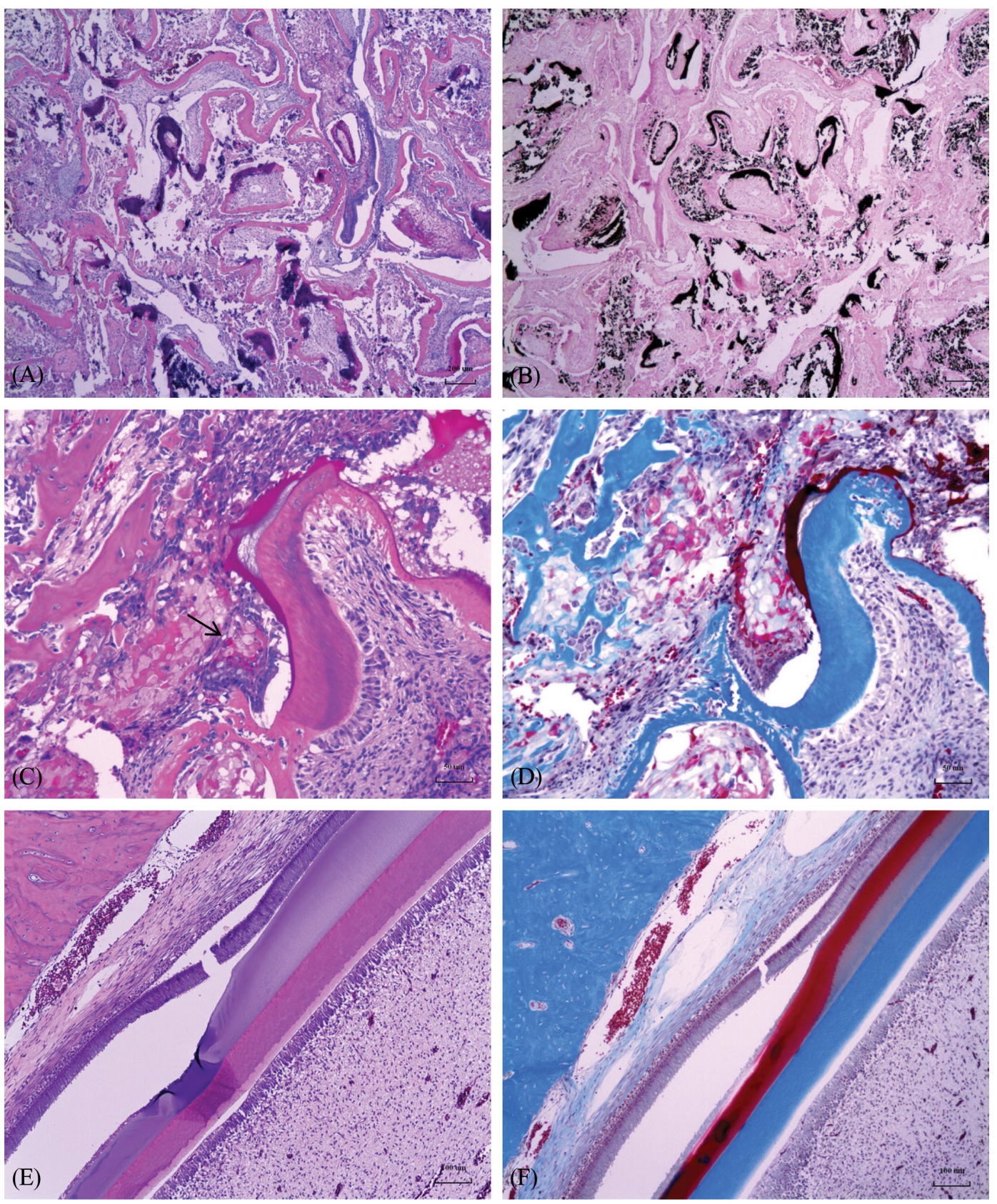

Fig. 3. (A) H\&E staining. The hard tissue matrix in the center of the tumor was basophilic when calcified, and eosinophilic when uncalcified. Scale bar $=200 \mu \mathrm{m}$. (B) von Kossa staining. Only hard tissue areas that were basophilic with H\&E stain appeared positive for calcium (black) with von Kossa stain. Scale bar $=200 \mu \mathrm{m}$. (C) H\&E staining. Abortive tooth-like structures and areas of ossification, osseous and squamous metaplasia (arrow) were present in the tumor. Scale bar $=50 \mu \mathrm{m}$. (D) Masson's Trichrome staining. Eosinophilic matrix and ossification tissue (collagenous tissues) appeared blue with Masson's trichrome stain. The deeply eosinophilic matrix, squamous metaplasia, and eosinophilic granular cells stained with H\&E appeared dark red with Masson's Trichrome stain. Scale bar= $50 \mu \mathrm{m}$. (E) H\&E staining. Normal incisor tooth was appeared. Scale bar $=100 \mu \mathrm{m}$. (F) Masson's Trichrome staining. In normal teeth, dentin, bone, and fibrous tissue appeared blue and the enamel matrix appeared dark red. Scale bar $=100 \mu \mathrm{m}$. 

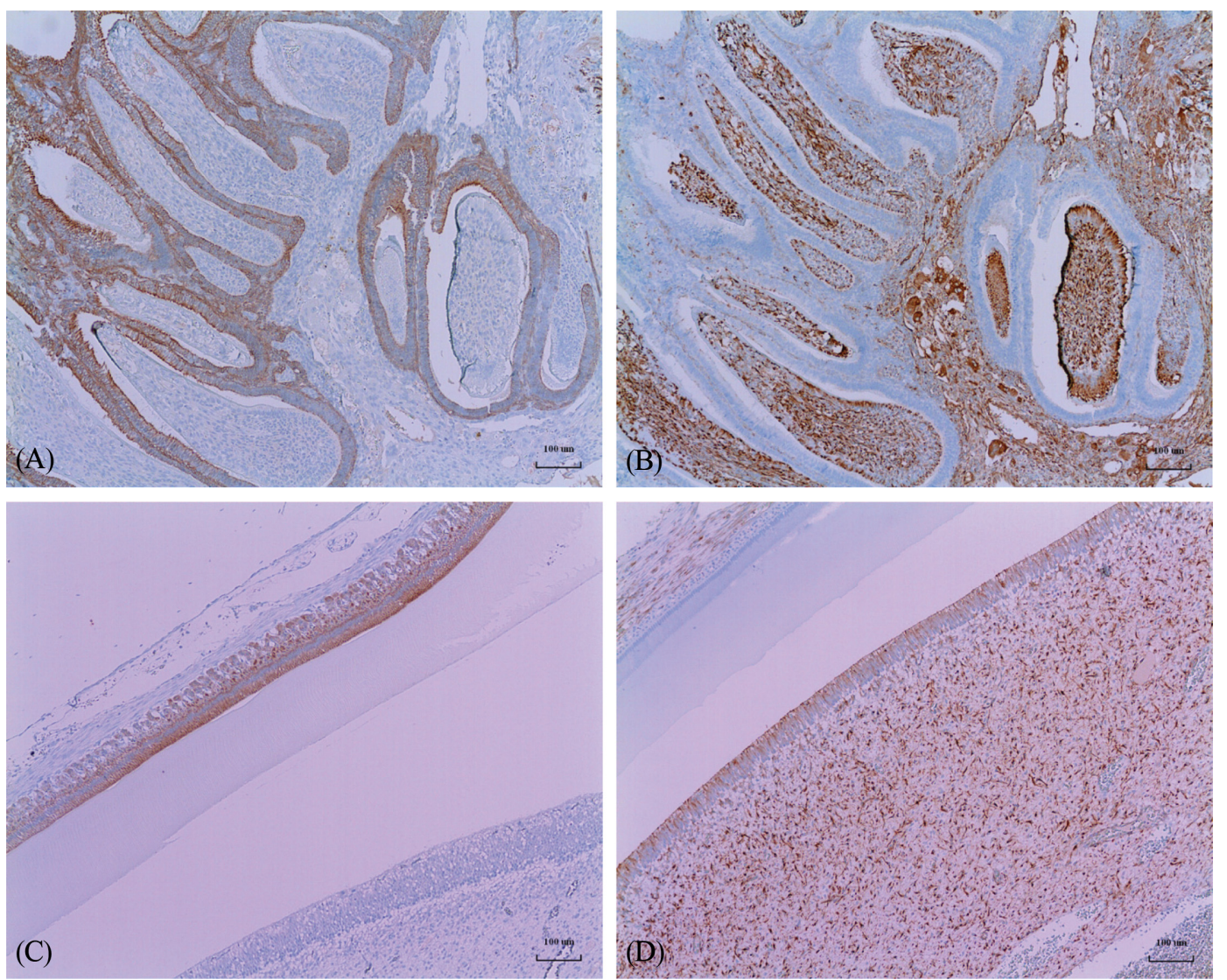

Fig. 4. Immunohistochemical staining. (A) The epithelial cell layer was severe positive for CK AE1/AE3. Scale bar $=100 \mu \mathrm{m}$. (B) The surrounding mesenchymal cells, odontoblast-like cells, and satellite reticulum-like cells were severe positive for vimentin. Scale bar = $100 \mu \mathrm{m}$. (C) In normal tooth, the ameloblasts were severe positive for the CK AE1/AE3. Scale bar $=100 \mu \mathrm{m}$. (D) In normal tooth, the dental pulp and odontoblasts were severe positive for vimentin. Scale bar $=100 \mu \mathrm{m}$.

As the dental tissue was likely to resemble bone tissue, ossification or osseous metaplasia may indeed occur (Fig. 3C). In some areas, deeply eosinophilic matrix was present adjacent to the layers of dentin (Fig. 3C).

Cystic degeneration was seen in the tumor (Fig. 2A). Mitotic figures were rare and no tumor invasion of the surrounding tissue was seen.

Histochemically, only the calcified part of the hard tissue and bone were stained black with von Kossa staining in undecalcified sections (Fig. 3B). The eosinophilic or basophilic matrix and bone tissue were stained blue with Masson's Trichrome staining (Fig. 3D), which was consistent with collagen, the major organic component of dentin. The deeply eosinophilic matrix, squamous metaplasia, and granular cytoplasmic cells were stained dark red with Masson's Trichrome staining (Fig. 3D), which was consistent with keratin. The PAS reaction in the squamous metaplastic tissue and eosinophilic granular cells was variable, showing little or no glycogen. In the normal tooth, the enamel matrix was stained dark red and the dentin and bone tissue were stained blue with Masson's Trichrome staining (Fig. 3E, 3F).
The palisading columnar cells were positive for CK AE1/ AE3 (Fig. 4A) and negative for vimentin, whereas the surrounding mesenchymal component and intracavity islands, such as mesenchymal cells, stellate reticulum-like cells, odontoblast-like cells, were positive for vimentin (Fig. 4B) and negative for CK AE1/AE3. Also, the ameloblasts in normal tooth were severe positive for CK AE1/AE3 (Fig. 4C) and negative for vimentin, whereas the dental pulp and odontoblast were severe positive for vimentin (Fig. 4D) and negative for CK AE1/AE3.

\section{DISCUSSION}

The diagnosis of ameloblastic odontoma was based on: 1) irregular cords with follicles of odontogenic epithelium abutting a small cluster of odontoblasts resembling the enamel structure in the developing tooth germ and 2) formation of osteodentin $(1,11)$.

In our case, the histological figures showed odontogenic epithelium, odontogenic ectomesenchyme, enamel formation, and mineralized dental tissues, including dentin, osteoden- 
tin, and bone-like tissue. The tumor showed a biphasic growth consisting of epithelial and mesenchymal components. Ameloblast-like cuboidal epithelial cells were present in the outer areas of dental structures.

Immunohistochemical staining revealed that the epithelial components of the tumor were CK AE1/AE3 severe positive and vimentin negative, whereas the surrounding mesenchymal components were $\mathrm{CK}$ AE1/AE3 negative and vimentin severe positive. In normal tooth, the ameloblasts were CK AE1/AE3 positive and vimentin negative, whereas the dental pulp and odontoblasts were CK AE1/AE3 negative and vimentin positive. These results indicate that the epithelial components originated from the odontogenic epithelium and that the mesenchymal components originated from the odontogenic ectomesenchyme.

The histologic and histochemical features of the hard tissue in the ameloblastoma were most consistent with enamel, dentin, and osteodentin. Osteodentin is rapidly formed, tertiary dentin that often entraps odontoblasts with few cognizable dentin tubules, morphologically similar to bone in appearance (19). The formation of metaplastic osteodentin in the pulp cavity of rat incisors was a well-recognized reaction in toxicologic studies (18). The proliferating ameloblasts were accompanied by several apparent inductive changes in the surrounding stellate reticulum. The most prominent was deposition of bone-like tissue that contributed to the bulk of the mass (9). The combination of predominant ameloblastoma with features of odontogenic ectomesenchyme and mineralized dental tissues in a single lesion thus warrants the diagnosis of ameloblastic odontoma $(2,3)$.

In the rat, lesions arising from the mandible with features of odontogenic epithelium and mineralized dental tissues have been reported as either spontaneous $(2,12)$ or chemically induced tumors (13) and were diagnosed as ameloblastic odontoma.

A malignant odontogenic tumor that metastasized to multiple distal organs has been reported in a dog (20). Morphologic criteria for malignancy were pleomorphism, layering of secretory epithelium, invasion of surrounding tissue, high mitotic activity, anaplasia, and metastases (21). In our case, relatively slow growth, scarce mitotic figures, no tumor invasion in the surrounding tissue, and no distant metastasis were seen. Therefore, the present case was diagnosed as benign ameloblastic odontoma.

Furthermore, our study revealed biphasic neoplasia in the odontogenic epithelium and odontogenic mesenchyme, although connective tissue formation was relatively scanty in the entire tumor. Accordingly, based on the nomenclature of odontogenic tumors in animals (22), we have chosen to use the term "ameloblastic odontoma" for this tumor which included both ameloblastoma and odontoma.

Peripheral odontogenic tumor has been reported at unusual sites including the buccal mucosa (23), base of tongue (24), floor of the mouth (25), and mandibular lingual gingiva
(26). However, the origin and nature of peripheral odontogenic tumor have not been clearly established. Potential sources include odontogenic remnants of the vestibular lamina, pluripotent cells in the basal cell layer of the mucosal epithelium, and pluripotent cells of the salivary glands $(17,18)$.

Ida-Yonemochi et al. hypothesized that the dental follicle or the periodontal ligament contain cytokines specific for osteoclastic differentiation and then the osteoclast might be closely associated with tooth germ development (27). In our study, we used conventional TRAP histochemistry to identify osteoclasts. We confirmed that many osteoclasts were present along the osseous tissue in this tumor. Pekiner et al. reported that bone changes were seldom present in peripheral ameloblastoma (16). A few cases of odontogenic tumor have been reported in which the tumors have arisen in the soft tissues without bone involvement, implying origin from remnants of the dental lamina (28). The theories of pathogenesis have been especially well reviewed by Hertz (29). Histologically, the extraosseous location was the peculiar feature of peripheral odontogenic tumor, which was otherwise similar to the classical intraosseous odontogenic tumor $(15,16)$.

In our case, the tumor did not appear to attach to the mandibular bone or to be continuous with the normal teeth, oral cavity epithelium, and salivary gland or duct. The tumor was dissected totally free by blunt dissection with relative ease. Histopathologically, the tumor was combined ameloblastoma with features of odontogenic ectomesenchyme and composite odontoma, usually resembling the intraosseous origin of ameloblastic odontoma (2-4). Therefore, we could suggest the tumor did not arise from the intraosseous region, mucosal epithelium, or salivary glands. Therefore, the tumor was probably derived from odontogenic epithelial remnants in an extraosseous region.

In conclusion, on the basis of clinical sign, anatomical location, necropsy finding, histomorphology, and immunohistochemical characteristics, the present case was diagnosed as peripheral ameloblastic odontoma. We hope that this report will contribute to the classification of this uncommon tumor in rodents.

\section{ACKNOWLEDGMENTS}

The authors would like to thank the technicians at the Department of Pathology of Chemon Inc. for technical assistance. This work was fully supported by Chemon Inc.

\section{REFERENCES}

1. Kramer, I.R.H., Pindborg, J.J. and Shear, M. (1992) World Health Organization (International histological classification of tumours). Histological typing of odontogenic tumours (2nd edition), Springer-Verlag Heidelberg, New York. 
2. Burrough, E.R., Myers, R.K. and Whitley, E.M. (2010) Spontaneous odontoameloblastoma in a female Sprague Dawley rat. J. Vet. Diagn. Invest., 22, 998-1001.

3. Mosqueda-Taylor, A., Carlos-Bregni, R., Ramirez-Amador, V., Palma-Guzman, J.M., Esquivel-Bonilla, D. and Hernandez-Rojase, L.A. (2002) Odontoameloblastoma. Clinic-pathologic study of three cases and critical review of the literature. Oral Oncol., 38, 800-805.

4. Weber, K. (2007) Induced and spontaneous lesions in teeth of laboratory animals. J. Toxicol. Pathol., 20, 203-213.

5. Nold, J.B., Powers, B.E., Eden, E.L. and McChesney, A.E. (1984) Ameloblastic odontoma in a dog. J. Am. Vet. Med. Assoc., 185, 996-998.

6. Quigley, P.J. and Leedale, A.H. (1983) Tumors involving bone in the domestic cat: A review of fifty-eight cases. Vet. Pathol., 20, 670-686.

7. Chalmers, G.A. and Shacklady, E.T. (1991) Ameloblastic odontoma in a calf. Can. Vet. J., 32, 365-366.

8. Dubielzig, R.R. and Griffith, J.W. (1982) An odontoameloblastoma in an adult sheep. Vet. Pathol., 19, 318-320.

9. Step, D.L., Ritchey, J.W., Drost, W.T. and Bahr, R.J. (2003) Ameloblastic odontoma in the mandible of a llama. Can. Vet. $J .$, 44, 824-827.

10. Davis, J.A., Banks, R.E. and Young, D. (1988) Ameloblsatic odontoma in a cynomolgus monkey (Macaca fascicularis). Lab. Anim. Sci., 38, 312-315.

11. Yanai, T., Masegi, T., Tomita, A., Kudo, T., Yamazoe, K., Iwasaki, T., Kimura, N., Katou, A., Kotera, S. and Ueda, K. (1995) Odontoameloblastoma in a Japanese Monkey (Macaca fuscata). Vet. Pathol., 32, 57-59.

12. Fitzgerald, J.E. (1987) Ameloblastic odontoma in the Wistar rat. Toxicol. Pathol., 15, 479-481.

13. Wang, H., Terashi, S. and Fukunishi, R. (1975) Ameloblastic odontoma in rats induce by N-butylnitrosourea. Gan, 66, 319321 .

14. Stanley, H.R.J. and Krogh, H.W. (1959) Peripheral ameloblastoma: Report of a case. Oral Surg. Oral Med. Oral Pathol., 12, 760-765.

15. Wettan, H.L., Patella, P.A. and Freedman, P.D. (2001) Peripheral ameloblastoma: review of the literature and report of recurrence as severe dysplasia. J. Oral Maxillofac. Surg., 59, 811-815.

16. Pekiner, F.N., Ozbayrak, S., Sener, B.C., Olgac, V. and Sinanoglu, A. (2007) Peripheral ameloblastoma: a case report.
Dentomaxillofac. Radiol., 36, 183-186.

17. Kishino, M., Murakami, S., Yuki, M., Iida, S., Ogawa, Y., Kogo, M. and Toyosawa, S. (2007) A immunohistochemical study of the peripheral ameloblastoma. Oral. Dis., 13, 575580 .

18. Kuijpers, M.H., van de Kooij, A.J. and Slootweg, P.J. (1996) Review article. The rat incisor in toxicologic patholody. Toxicol. Pathol., 24, 346-360.

19. Karim, A.C. and Eddy, E.L. (1984) A light and electron microscopic study of osteodentin formation in the rat incisor after adriamycin administration. Am. J. Anat., 169, 207-219.

20. Ueki, H., Sumi, A., Takaishi, H., Ito, H., Oyamada, T. and Yoshikawa, H. (2004) Malignant ameloblastic fibro-odontoma in a dog. Vet. Pathol., 41, 183-185.

21. Stoica, G. and Koestner, A. (1984) Diverse spectrum of tumors in male Sparague-Dawley rats following single high doses of N-ethyl-N-nitrosourea (ENU). Am. J. Pathol., 116, 319-326.

22. Gardner, D.G. (1992) An orderly approach to the study of odontoenic tumours in animals. J. Comp. Pathol., 107, 427438.

23. Isomura, E.T., Okura, M., Ishimoto, S., Yamada, C., Ono, Y., Kishino, M. and Kogo, M. (2009) Case report of extragingival peripheral ameloblastoma in buccal mucosa. Oral Surg. Oral Med. Oral Pathol. Oral Radiol. Endod., 108, 577-579.

24. Rajesh, B.C., Vinayakumar, A.R., Mathai, J., Valsaladevi, S. and Nair, P.P. (1998) Peripheral ameloblastoma involving tongue-A rare case. Indian. J. Otolaryngol. Head Neck Surg., 50, 387-389.

25. Ramnarayan, K., Nayak, R.G. and Kavalam, A.G. (1985) Peripheral ameloblastoma. Int. J. Oral Surg., 14, 300-301.

26. Beena, V.T., Choudhary, K., Heera, R., Rajeev, R., Sivakumar, R. and Vidhyadharan, K. (2012) Peripheral ameloblastoma: A case report and review of literature. Case Rep. Dent., 2012, 571509.

27. Ida-Yonemochi, H., Ishibashi, O., Sakai, H. and Saku, T. (2004) Recruitment of osteoclasts in the mandible of osteopetrotic (op/op) mice. Eur. J. Oral. Sci., 112, 148-155.

28. Gorlin, R.J. and Meskin, L.H. (1963) Odontogenic tumors in man and animals: pathologic classification and clinical behavior-A review. Ann. N. Y. Acad. Sci., 108, 722-771.

29. Hertz, J. (1952) Adamantinoma; histo-pathologic and prognostic studies. Acta Chir. Scand., 102, 405-432. 